\title{
Interaction of the Sonic Boom with Atmospheric Turbulence
}

\author{
Zvi Rusak \\ Mechanical Engineering, Aeronautical Engineering and Mechanics, \\ Julian D. Cole \\ Department of Mathematical Sciences, \\ Rensselaer Polytechnic Institute
}

Troy, NY $12180-3590$

\begin{abstract}
Theoretical research has been carried out to study the effect of free-stream turbulence on sonic boom pressure fields. A new transonic small-disturbance model to analyze the interactions of random disturbances with a weak shock has been developed. The model equation has an extended form of the classic small-disturbance equation for unsteady transonic aerodynamics. An alternative approach shows that the pressure field may be described by an equation that has an extended form of the classic nonlinear acoustics equation that describes the propagation of sound beams with narrow angular spectrum. The model shows that diffraction effects, nonlinear steepening effects, focusing and caustic effects and random induced vorticity fluctuations interact simultaneously to determine the development of the shock wave in space and time and the pressure field behind it. A finite-difference algorithm to solve the mixed-type elliptic-hyperbolic flows around the shock wave has also been developed. Numerical calculations of shock wave interactions with various deterministic and random fluctuations will be presented in a future report.
\end{abstract}




\section{Contents}

1 Introduction 1

2 The Breakdown of the Linearized Theory 5

2.1 The Linearized Theory . . . . . . . . . . . . . . 5

2.2 One Dimensional Flow . . . . . . . . . . . . . 8

3 A Transonic Small Disturbance Model - 11

4 Finite Difference Scheme 16

5 Conclusions $\quad 20$ 


\section{Introduction}

Experimental data exists showing the pressure profiles of sonic booms, created by the passage of a distant supersonic aircraft, can be drastically affected by free-stream atmospheric turbulence. ${ }^{1-5}$ The usual $N$-wave or a shaped sonic boom profile can randomly exhibit either large pressure peaks with short rise times or rounded profiles with longer rise times or messy pressure signatures. Recent laboratory model experiments to study the effect of turbulence on the rise time and wave form of $N$-waves have shown similar results. ${ }^{6}$ The interaction of the sonic boom with the atmospheric turbulence, specifically in the atmospheric boundary layer relatively near the ground, may result sometimes in higher, and may be unacceptable loudness levels. ' Therefore, in order to get reasonable estimates of the sonic boom performance of various designs of a future supersonic transport airplane it is essential to understand the basic interactions of the atmospheric turbulence with shock waves.

The basic analysis of the distortion of sonic bangs by atmospheric turbulence was given by $\mathrm{Crow}^{8}$ Using a first-order acoustic scattering theory, Crow showed that the pressure perturbation behind the shock is related to the interaction of the shock with the disturbances it encounters while moving in the atmosphere. The pressure profile can be calculated by a surface integral over a paraboloid of dependence, whose focus is the observation point and whose directix is the shock front. By describing the turbulent eddies in the Kolmogorov inertial subrange. it was found that the meansquare pressure perturbation behind the shock changes like $(\Delta p)^{2}\left(t_{c} / t\right)^{7 / 6}$ where $(\Delta p)$ is the pressure jump across the shock, $(t)$ is time after the shock passes an observation point and $\left(t_{c}\right)$ is a critical time predicted in terms of meteorological conditions. Crow's analysis predicts reasonable average values of the pressure fluctuations for times $(t)$ comparable to $\left(t_{c}\right)$.

The singularity in the pressure perturbations near the shock front (when $t \rightarrow 0$ ) was analyzed by Plotkin and George ${ }^{9}$. A second-order acoustic scattering theory was used to describe shock rounded signatures. The average of the diffraction effects was approximated as a dissipation term. 
The thickening of the shock is explained as a balance between nonlinear steepening effects and the dissipative effect of the turbulent scattering of acoustic energy out of the incident shock. Rise time predictions of this theory show some correlation with experimental data. On the other hand, Ffowcs Williams and Howe ${ }^{10}$ examined the approaches that describe the possibility of a turbulent thickening of weak shock waves and reached a conclusion that atmospheric turbulence cannot be the cause of shock thickening. They suggested that weak shocks may attain a dispersed profile due to non-equilibrium gas effects.

It should be emphasized here that the scattering analyses of References 8-10 considered small turbulent perturbations against the shock strength, whereas in the case of the interaction of the sonic boom with atmospheric turbulence the flow random fluctuations may be of the same order of the shock weak strength and may strongly distort the shock front. The above analyses also did not account for shock jump conditions that must be satisfied in an inviscid analysis across any shock surface. The approximation made in Reference 9 of the average diffraction effects described as a dissipative term is also unclear.

A different approach was taken by Pierce ${ }^{11.12}$. He interpreted the spikes observed on sonic boom pressure waveforms as being due to the simultaneous focusing and diffraction of a nearly planar. $V$-wave by an inhomogeneous layer in the atmosphere. The shock front develops ripples that are transformed into folds in the front when the shock passes vertices of caustics. This mechanism results in a fine structure of very small pressure jumps that correspond to the various segments of the folded wavefront. Pierce ${ }^{12}$ derived a stochastic model of a sharp shock propagating through a turbulent atmosphere to substantiate the very small discrete structure of sonic boom profiles.

Pierce model ${ }^{11.12}$, however, neglects nonlinear effects that become significant specifically near a caustic vertex as was shown by Cramer and Seebass ${ }^{13}$ and Gill and Seebass ${ }^{14}$. Cramer and Seebass ${ }^{13}$ described the focusing of a very weak and slightly concave shock wave by the unsteady transonic small-disturbance flow equation. Gill and Seebass ${ }^{14}$ derived an 
approximate analytical solution of the steady transonic small-disturbance problem for the nonlinear behavior of a weak compression wave with a finite rise time near a caustic. They calculated the reflected shock wave from a caustic and provided an estimate of its strength. The experimental results of Sturtevant and Kulkarny ${ }^{15}$ also show that focusing effects are specifically significant for weak shock waves as occurs in the case of the sonic boom signatures.

Sparrow and Pierce ${ }^{15}$ have recently presented a simple statistical prediction for how often sonic booms propagating in the earth's boundary layer will encounter caustics. The theory is based on describing the variation of ray tube areas of a sound wave propagating in a turbulent medium by a generic harmonic oscillator equation. For realistic realizations of atmospheric turbulence the model predicts that sonic booms will exhibit spikes with the occurrence of caustics after a very short distance of travel in the random medium, thus agreeing with the predictions of Pierce ${ }^{11,12}$.

In a recent paper, Pierce ${ }^{17}$ has derived a model equation to describe the development of sonic boom signatures in an atmospheric turbulence. The equation has been constructed by suing logical physical considerations only. It extends geometrical acoustic approximations to include convection at the wave speed, diffraction effects, molecular relaxation, classical dissipation and nonlinear steepening effects. The atmospheric turbulence enters through an effective speed of sound which varies randomly in time and space. However, since this theory has not been developed consistently from the fluid dynamic equations, Pierce ${ }^{17}$ raised questions whether all the effects are necessary in his suggested model and how to accomplish a numerical or analytical solution to the problem.

Related with the problem of the sonic boom interaction with turbulence is the basic question of the interaction of a shock wave with a vortical flow and specifically with a vortex or a train of eddies ${ }^{18-21}$. The research of the later problem was basically motivated by the interest to reduce the noise and vibrations produced by high-speed supersonic vehicles. For these problems, the interaction of relatively strong shock waves with turbulent jets or wakes is a significant source of noise. The shock-vortex system is a 
basic element of these more complex interactions ${ }^{21}$. It can also shed light on the sonic boom interaction with atmospheric turbulence, specifically when the shocks are weak anc vortex strength is comparable with the shock strength.

Experimental results of shock-vortex systems ${ }^{18-21}$ revealed curved, diffracted shocks as well as complicate structures of reflected curved shock waves from the incident shock front due to the vortex induced flow field. The pressure field behind the shock contains regions of compressions and rarefactions that produce acoustic waves. Similar shock structures were also observed by Sturtevant and Iiulkarny ${ }^{15}$ who investigated the focusing of weak curved shock waves. Of specific interest are Dosanjts and Weeks ${ }^{19}$ measurements of the interaction of a shock wave with a vortex street. The shock front is distorted by the wake flow which probably results in a focusing process, while the vortex street is rapidly dissolved by the shock.

The analyses of the shock-vortex interaction are limited to linear perturbation theories only. ${ }^{22-26}$ These analyses considered the jump conditions across a shock surface and predicted the development of vorticity waves, entropy waves and acoustic waves behind the shock front. ${ }^{22-24}$ The acoustic wave was approximated by a quadrupolar ${ }^{26}$ or as a sum of monopole, dipole and quadrupole acoustic sources. However, since all of these theories are linear, they cannot account for any nonlinear effects due to shock large distortions, focusing and caustic effects or nonlinear steepening effects that are found in experiments ${ }^{18-20}$ or in recent numerical simulations of shock-vortex interactions. ${ }^{2 i-29}$

The review of experimental and theoretical investigations of the interaction of shock waves with free stream vortical or turbulent flows shows that this complex nonlinear interaction is still an open problem. ${ }^{27}$ Specifically, the improved simulation of sonic boom propagation through the real atmosphere requires a better understanding of the interaction of sonic boom with atmospheric turbulence. ${ }^{16}$

The analysis of the experimental data and the theoretical approaches shows that in the case of the sonic boom, the shock waves near ground 
are very weak, but still stronger than any acoustic wave. Also, flow fluctuations due the atmospheric turbulence can become comparable to the shock weak strength such that locally the shock strength can be strongly reduced or magnified and the shock wave front can be distorted significantly. Therefore, linearized acoustics and its second-order scattering problem, or first-order linear theories of shock-vorticity interaction do not represent correctly the development of the weak shock and the pressure field behind it (see also Section 2). However, in a coordinate system moving with the basic weak shock, the problem may fit the transonic framework.

This paper presents a new transonic small-disturbance model that has been developed to describe the interactions of randon fluctuations with a weak shock wave. The model equation is found to have an extended form of the classic nonlinear acoustics equation that describes the propagation of sound beams with narrow angular spectrum (KKZ equation). ${ }^{30,31}$ The model shows that diffraction effects. nonlinear steepening effects, focusing and caustic effects and random induced vorticity fluctuations interact simultaneously to determine the development of the shock wave in space and time and the pressure field behind it. Af finite-difference algorithm to solve the mixed-type elliptic-hyperbolic flows around the shock wave is also presented. The results of the numerical calculations will be presented in a future report. It is expected to find solutions that will describe both peaked or rounded or messy pressure signatures as were recorded in experiments.

\section{The Breakdown of the Linearized Theory}

\subsection{The Linearized Theory}

An inviscid and a non heat conducting flow is assumed. A normal shock with a uniform supersonic oncoming stream and a uniform subsonic outgoing flow is considered. The upstream flow ahead of he shock is characterized by a speed $\left(U_{0 a}\right)$ in the $x$-direction, pressure $\left(p_{0 a}\right)$ and density $\left(\rho_{0 a}\right)$ and

the downstream flow behind the shock by $\left(V_{0 b}\right),\left(p_{0 b}\right)$ and $\left(\rho_{0 b}\right)$ respectively. 
Assuming the shock front is given by the $x=0$ plane, the jump conditions across a normal shock ${ }^{32}$ show that:

$$
\begin{aligned}
\rho_{0 a} U_{0 a} & =\rho_{0 b} U_{0 b} \\
\rho_{0 a} U_{0 a}^{2}+p_{0 a} & =\rho_{0 b} U_{0 b}^{2}+p_{0 b} \\
\frac{1}{2} \rho_{0 a} U_{0 a}^{3}+\frac{\gamma}{\gamma-1} p_{0 a} U_{0 a} & =\frac{1}{2} \rho_{0 b} U_{0 b}^{3}+\frac{\gamma}{\gamma-1} p_{0 b} U_{0 b}
\end{aligned}
$$

Small disturbances are considered in each of the uniform streams. The velocity vector $(V)$. pressure $(P)$, density $(\rho)$ and vorticity $(\underset{\sim}{\omega})$ are given ahead $(j=a)$ and behind the shock $(j=b)$ by:

$$
\begin{aligned}
& \underline{\sim}_{j}=l_{0 j}\left({\underset{\sim}{I}}_{x_{1 j}}+\epsilon v_{v_{1 j}}+\cdots\right) \\
& P_{3}=P_{0,}\left(1+\epsilon p_{1},+\cdots\right) \\
& \rho_{j}=\rho_{0 j}\left(1+\epsilon \rho_{1},+\cdots\right) \\
& {\underset{\sim}{\omega},}_{j}=\epsilon{\underset{\sim}{1},}_{1}+\cdots, \quad{\underset{\sim}{1 j}}_{j}=\nabla \times{\underset{\sim}{v}, j}^{v}
\end{aligned}
$$

Here ${\underset{\sim}{v j}}_{1 j}, p_{1 j}, \rho_{1 j}$ are functions of $(x, y, z, t)$. An axial coordinate moving with the uniform speed is considered in each region, $\xi_{j}=x-U_{0,} t, \tau=$ $U_{0} t$. The substitution into the continuity, momentum and energy equations results to the leading order in $\left(\underset{\sim}{\nabla}=\frac{\partial}{\partial \xi_{1}} \sim_{x}+\frac{\partial}{\partial y} e_{\sim_{y}}+\frac{\partial}{\partial z} \underset{\sim_{z}}{e}\right)$ :

$$
\begin{array}{r}
\frac{\partial \rho_{1 j}}{\partial \tau}+\underset{\sim}{\nabla}{\underset{\sim}{\sim 1 j}}_{v}^{v}=0 \\
\frac{\partial v_{\sim_{j}}^{v}}{\partial \tau}+\frac{1}{\gamma M_{0 j}^{2}} \underset{\sim}{\nabla} p_{1 j}=0
\end{array}
$$




$$
\frac{\partial p_{1 j}}{\partial \tau}+\gamma\left(\underset{\sim}{\nabla} \underset{\sim}{u_{1}}\right)=0
$$

Equations (3) result in:

$$
\frac{\partial p_{1 j}}{\partial \tau}=\gamma \frac{\partial \rho_{1,}}{\partial \tau}, \quad \frac{\partial \omega_{1 j}}{\partial \tau}=0 . \quad W_{0 j}^{2} \frac{\partial^{2} p_{1 j}}{\partial \tau^{2}}-\nabla^{2} p_{1,}=0
$$

where $M_{0 j}=U_{0 j} / c_{0 j}$ and $c_{0_{j}}^{2}=\gamma p_{0 j} / \rho_{0 j}$. Equations (4) show that ${\underset{\sim}{l_{j}}}_{1}=$ ${\underset{\sim}{\omega}}_{1 j}\left(\xi_{j}, y, z\right)$ and that the pressure perturbation $p_{1}$ is described by the acoustics equation. Therefore, the first order disturbance flow can be split into a linear combination of rotational and irrotational parts: $v_{1 j}=\underline{\sim}_{1 j,}^{v}+v_{\sim 1,0}$. The rotational part can be described essentially by incompressible flow equations:

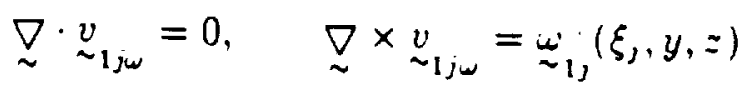

The irrotational (potential) part may be described by acoustics equations relative to the basic flow in each region:

$$
\stackrel{v}{\sim}_{1 j 0}=\nabla^{\prime} \phi_{j}, \quad p_{1 j}=-\gamma \cdot M_{0_{j}}^{2} \frac{\partial \phi_{j}}{\partial \tau}, \quad . M_{0 j}^{2} \frac{\partial^{2} \phi_{j}}{\partial \tau^{2}}-\nabla^{2} \phi_{j}=0
$$

The first-order perturbation theory also considers the distortion of the shock front. Assuming that the perturbed shock front is given by $x-$ $\epsilon g_{1}(y, z, t)=0$, the exact jump condition across the shock ${ }^{32}$ result to the leading order in a set of conditions that must be satisfied along the $x=0$ plane for any $(y, z, t)$ :

$$
\begin{gathered}
\rho_{1 a}(0, y, z, t)+u_{1 a}(0, y, z, t)-\frac{g_{1 t}}{U_{0 a}}=\rho_{1 b}(0, y, z, t)+u_{1 b}(0, y, z, t)-\frac{g_{1 t}}{U_{0 b}} \\
\begin{aligned}
\left.U_{0 a}\left(\rho_{1 a}(0, y, z, t)+2 u_{1 a}(0, y, z, t)\right)+\frac{1}{\gamma M_{0 a}^{2}} p_{1 a}(0, y, z, t)\right)= \\
=
\end{aligned} U_{0 b}\left(\rho_{1 b}(0, y, z, t)+2 u_{1 b}(0, y, z, t)+\frac{1}{\gamma M_{0 b}^{2}} p_{1 b}(0, y, z, t)\right)
\end{gathered}
$$




$$
\begin{aligned}
& U_{0 a}^{2}\left(\rho_{1 a}(0, y, z, t)\right.+3 u_{1 a}(0, y, z, t)+\frac{2}{(\gamma-1) \cdot M_{0 a}^{2}}\left(p_{1 a}(0, y, z, t)+\right. \\
&\left.\left.+u_{1 a}(0, y, z, t)\right)-\frac{g_{1 t}}{U_{0 a}}\left(1+\frac{2 / \gamma}{(\gamma-1) M_{0 a}^{2}}\right)\right) \\
&=U_{0 b}^{2}\left(\rho_{1 b}(0, y, z, t)+3 u_{1 b}(0, y, z, t)+\frac{2}{(\gamma-1) \cdot M_{0 b}^{2}}\left(p_{1 b}(0, y, z, t)+\right.\right. \\
&\left.\left.+u_{1 b}(0, y, z, t)\right)-\frac{g_{1 t}}{U_{0 b}}\left(1+\frac{2 / \gamma}{(\gamma-1) M_{0 b}^{2}}\right)\right) \\
& U_{0 a}\left(v_{1 a}(0, y, z, t)+g_{1 y}\right)=U_{0 b}\left(v_{1 b}(0, y, z, t)+g_{1 y}\right) \\
& U_{0 a}\left(w_{1 a}(0, y, z, t)+g_{1 z}\right)=U_{0 b}\left(u_{1 b}(0, y, z, t)+g_{1 z}\right)
\end{aligned}
$$

Here $g_{1 t}=\partial g_{1} / \partial t$ and $\left(u_{1}, v_{1}, u_{1}\right)$ are the components of the velocity perturbation $\underset{\sim 1}{v}$. The linearized jump conditions in Eqs. (6) include the entropy increase produced by the shock. It can be shown from Eqs. (5) and (6) that, using the solution of the downstream equations for the disturbance flow, the shock conditions are adequate to describe the flow downstream and the disturbed motion of the shock wave for given upstream disturbances.

\subsection{One Dimensional Flow}

In the case of a one-dimensional flow, the rotational part vanishes identically and the solution of the acoustics equation ahead $(j=a)$ and behind $(j=b)$ 
the shock is given by:

$$
\begin{aligned}
& u_{1 j}=F\left(\xi_{j}-\frac{T}{M_{0 j}}\right)+G\left(\xi_{j}+\frac{T}{M_{0 j}}\right) \\
& p_{1 j}=\gamma M_{0 j}\left(F\left(\xi_{j}-\frac{\tau}{M_{0 j}}\right)-G\left(\xi_{j}+\frac{\tau}{M_{0 j}}\right)\right) \\
& \rho_{1 j}=M_{0_{j}}\left(F\left(\xi_{j}-\frac{\tau}{. M_{0 j}}\right)-G\left(\xi_{j}+\frac{\tau}{M_{0_{j}}}\right)+\rho_{s_{j}}\left(\xi_{\jmath}\right)\right)
\end{aligned}
$$

where $F$ and $G$ are arbitrary functions that describe the upstream and downstream acoustic waves. Here $\rho$, is an arbitrary function that describes entropy waves that are convected with the flows (the entropy first-order disturbance is given by $S_{j}-S_{0 j}=\epsilon s_{1}=-\epsilon c_{p} M_{0,} \rho_{s j}\left(\xi_{j}\right)$, where $S_{j}$ is the entropy in region $j, S_{0}=c_{v} \ln \left(P_{0} / \rho_{0}^{\gamma}\right)$ and $c_{v}, c_{p}$ are the specific heat constants). Assuming that no upstream acoustic waves can develop, specifically not in the flow behind the shock then $G \equiv 0$. Then the shock jump conditions (6) provide a system of 3 linear equations for the solution of the downstream acoustic and entropy waves $F_{b}\left(\xi_{b}-\frac{r}{M_{0 b}}\right)$ and $\rho_{s b}\left(\xi_{b}\right)$ and the shock position rate of change in time $g_{1 t}(t)$ in terms of the given acoustic and entropy perturbations $F_{a}\left(\xi_{a}-\frac{r}{V_{0 a}}\right)$ and $\rho_{s a}\left(\xi_{a}\right)$ in the upstream flow. Let,

$$
\begin{array}{ll}
F_{a 0}=F_{a}\left(-\left(U_{0 a}+c_{0 a}\right) t\right), & \rho_{s a 0}=\rho_{s a}\left(-U_{0 a} t\right) \\
F_{b 0}=F_{b}\left(-\left(U_{0 b}+c_{0 b}\right) t\right) . & \rho_{s b 0}=\rho_{s b}\left(-U_{0 b} t\right)
\end{array}
$$

then from Eqs. (6) - (8) we get: 


$$
\begin{gathered}
\left(1+M_{0 b}\right) F_{b 0}+M_{0 b} \rho_{s b 0}+\left(\frac{U_{0 b}}{U_{0 a}}-1\right) \frac{g_{1 b}}{U_{0 b}}=\left(1+M_{0 a}\right) F_{a 0}+M_{0 a} \rho_{s a 0} \\
\left(2+M_{0 b}+\frac{1}{V_{0 b}}\right) F_{b 0}+M_{0 b} \rho_{s b 0}=\frac{U_{0 a}}{U_{0 b}}\left(\left(2+. M_{0 a}+\frac{1}{M_{0 a}}\right) F_{a 0}+M_{0 a} \rho_{s a 0}\right) \\
\left(3+M_{0 b}+\frac{2}{(\gamma-1) \cdot M_{0 b}^{2}}\left(1+\gamma \cdot M_{0 b}\right)\right) F_{b 0}+M_{0 b} \rho_{s b 0}+ \\
+\left(\frac{U_{0 a}}{U_{0 b}}\left(1+\frac{2 / \gamma}{(\gamma-1) \cdot L_{0 a}^{2}}\right)-1-\frac{2 / \gamma}{(\gamma-1) \cdot V_{0 b}^{2}}\right) \frac{g_{1 t}}{U_{0 b}}= \\
=\frac{U_{0 a}^{2}}{U_{0 b}^{2}}\left(\left(3+M_{0 a}+\frac{2}{(\gamma-1) M_{0 a}^{2}}\left(1+\gamma M_{0 a}\right)\right) F_{a 0}+M_{0 a} \rho_{s a 0}\right)
\end{gathered}
$$

The determinant of the system ( 9 ) may be written in the form $\Delta=$ $\left(M_{0 a}^{2}-1\right) f n\left(. M_{0 a}^{2}\right)$. Therefore, the solution of Eqs. (9) shows that the shock front motion and the perturbed flow behind it may be described by:

$$
\begin{aligned}
x & =\frac{\epsilon}{M_{0 a}^{2}-1} \dot{g}_{1}\left(t ; M_{0 a}\right) \\
u_{1 b} & =U_{0 b}\left(1+\frac{\epsilon}{M_{0 a}^{2}-1} \bar{u}_{1}\left(x, t: M_{0 a}\right)+\cdots\right) \\
p_{1 b} & =P_{0 b}\left(1+\frac{\epsilon}{M_{0 a}^{2}-1} \tilde{p}_{1}\left(x, t ; M_{0 a}\right)+\cdots\right) \\
\rho_{1 b} & =\rho_{0 b}\left(1+\frac{\epsilon}{. M_{0 a}^{2}-1} \dot{\rho}_{1}\left(x, t:{U_{0 a}}_{0}\right)+\cdots\right)
\end{aligned}
$$

The functions $\dot{g}_{1}, \tilde{u}_{1}, \dot{p}_{1}, \dot{\rho}_{1}$ can be expressed in terms of the given flow perturbations ahead of the shock wave. In principle, these expressions may 
enable a spectral characterization of the pressure fluctuations and the turbulence downstream of the shock wave in terms of the spectral characterization of the incoming turbulence. However. Eqs. (10) show that the linear approach is a non-uniform approach when the shock wave is very weak $M_{0 a}^{2} \rightarrow 1^{+}$and the flow fluctuations are of the same order of the shock strength, $\epsilon \sim\left(M_{0 a}^{2}-1\right)$, as is the case of the interaction of the sonic boom with atmospheric turbulence. A similar nonuniformity is also expected from the analysis of two- or three-dimensional flows. However, this uniformity problem leads to a different approach to study the interaction of a weak shock with comparable random fluctuations in the flow.

\section{A Transonic Small Disturbance Model}

The analysis of the linearized problem of the interaction of a weak shock with small disturbances shows that it is an invalid approach when the flow perturbations are of the order of the shock strength. Therefore, a different approach has been developed to study the interaction of weak shocks with comparable random fluctuations in the flow. In a coordinate system moving with a basic given weak shock. the problem may fit the transonic theory framework. A transonic small-disturbance model is developed to analyze the flow across a basic weak shock running in the $(-x)$ direction. A coordinate system attached to the basic shock is considered. The velocity vector $(\underset{\sim}{V})$, pressure $(P)$, density $(\rho)$ and vorticity $(\underset{\sim}{\sim})$ are described every where in the flow by:

$$
\begin{aligned}
\underset{\sim}{V}= & U_{\infty}\left\{\underset{\sim}{i}\left(1+\epsilon^{2 / 3} u+\epsilon u_{1}+\epsilon^{t / 3} u_{2}+\cdots\right)\right. \\
& \left.+\underset{\sim}{j}\left(\epsilon v_{1}+\epsilon^{4 / 3} v_{2}+\cdots\right)+\underset{\sim}{k}\left(\epsilon w_{1}+\epsilon^{4 / 3} w_{2}+\cdots\right)\right\} \\
P= & p_{\infty}\left(1+\epsilon^{2 / 3} p+\epsilon p_{1}+\epsilon^{t / 3} p_{2}+\cdots\right) \\
\rho= & \rho_{\infty}\left(1+\epsilon^{2 / 3} \rho+\epsilon \rho_{1}+\epsilon^{4 / 3} \rho_{2}+\cdots\right)
\end{aligned}
$$




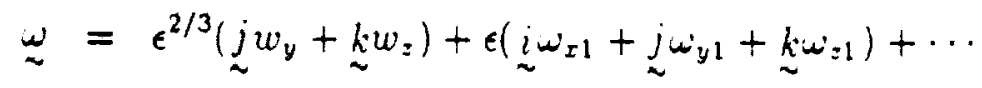

where $U_{\infty}=a_{\infty}\left(1+\frac{K}{2} \epsilon^{2 / 3}\right)$ is the speed of the basic shock $(K>0)$ and $a_{\infty}, p_{\infty}, \rho_{\infty}$ are the speed of sound, pressure and density of the unperturbed flow ahead of the shock. $\left(\epsilon^{2 / 3}\right)$ represents the scale of strength of the basic weak shock where $\epsilon \ll 1$. A rescaling of the $x$-coordinate and time $(t)$ has also been considered: $x^{*}=\frac{x}{e^{1 / 3}}$ and $t^{*}=t a_{x} \epsilon^{1 / 3}$, such that each of the terms in (11) is a function of $\left(x^{*}, y, z, t^{*}\right)$. The rescaling in $x$ means a stretching of the picture of the flow around the basic shock in order to capture the basic nonlinear effects that occur in the flow across the shock. The rescaling in time accounts for low-frequency unsteady perturbations in the flow. The constant $K$ reflects that the speed of the basic shock wave is a little higher than the speed of sound. ahead of the shock. The substitution of Eqs. (11) into the continuity, momentum and energy equations results to the leading orders in:

$$
\begin{aligned}
& \left\{\begin{array}{l}
\frac{\partial}{\partial x^{*}}(\rho+u)=0 \\
\frac{\partial}{\partial x^{*}}\left(\rho_{1}+u_{1}\right)=0 \\
\frac{\partial \rho}{\partial t^{*}}+\frac{\partial}{\partial x^{*}}\left(u_{2}+\rho_{2}+\rho u\right)+\frac{\partial v_{1}}{\partial y}+\frac{\partial u_{1}}{\partial z}=0
\end{array}\right. \\
& \left\{\begin{array}{l}
\frac{\partial}{\partial x^{*}}(\gamma u+p)=0 \\
\frac{\partial}{\partial x^{*}}\left(\gamma u_{1}+p_{1}\right)=0 \\
\frac{\partial u}{\partial t^{*}}+\frac{\partial u_{2}}{\partial x^{*}}+\left(u+\rho+K^{*}\right) \frac{\partial u}{\partial x^{*}}+\frac{1}{y} \frac{\partial p_{2}}{\partial x^{*}}=0
\end{array}\right.
\end{aligned}
$$




$$
\left\{\begin{array}{l}
\frac{\partial v_{1}}{\partial x^{*}}=-\frac{1}{\gamma} \frac{\partial p}{\partial y} \\
\frac{\partial u_{1}^{\prime}}{\partial x^{*}}=-\frac{1}{\gamma} \frac{\partial p}{\partial z} \\
\frac{\partial p}{\partial t^{*}}-\gamma \frac{\partial \rho}{\partial t^{*}}+\rho \frac{\partial p}{\partial x^{*}}-\gamma p \frac{\partial \rho}{\partial x^{*}}+\frac{\partial p_{2}}{\partial x^{*}}-\gamma \frac{\partial \rho_{2}}{\partial x^{*}}=0 \\
\frac{\partial \omega_{y}}{\partial x^{*}}=0, \quad \frac{\partial \omega_{z}}{\partial x^{*}}=0
\end{array}\right.
$$

From the equation of state and the definition of entropy it can be shown that the temperature $T_{m}$ and entropy $S$ are given by:

$$
\begin{aligned}
T_{m} & =T_{x}\left(1+\epsilon^{2 / 3} T+\cdots\right) \\
S & =S_{x}\left(1+\epsilon^{2 / 3} s+\cdots\right)
\end{aligned}
$$

where

$$
\begin{gathered}
T_{\infty}=p_{\infty} / R \rho_{x} . \quad S_{x}=c_{v} \ln \left(\frac{p_{\infty}}{\rho_{\infty}^{\gamma}}\right) \\
T=p-\rho, \quad s=\frac{c_{v}}{S_{x}}(p-\gamma \rho)
\end{gathered}
$$

Equations (12) through (1i) result in:

$$
\begin{gathered}
u+\rho=f\left(y, z t^{*}\right) \\
\gamma u+p=g\left(y, z^{*}\right) \\
-2 \frac{\partial u}{\partial t^{*}}+\left(-K^{*}-f+g-(;+1) u\right) \frac{\partial u}{\partial x^{*}}+\frac{\partial v_{1}}{\partial y}+\frac{\partial w_{1}}{\partial z}=-\frac{1}{\gamma} \frac{\partial g}{\partial t^{*}}
\end{gathered}
$$




$$
\begin{gathered}
\omega_{y}=\frac{\partial u}{\partial z}-\frac{\partial u_{1}}{\partial x^{*}}=\frac{1}{\gamma} \frac{\partial g}{\partial z} \\
-\omega_{z}=\frac{\partial u}{\partial y}-\frac{\partial v_{1}}{\partial x^{*}}=\frac{1}{\gamma} \frac{\partial g}{\partial y} \\
T=g-f-(\gamma-1) u, \quad s=\frac{c_{u}}{S_{x}}(g-\gamma f)
\end{gathered}
$$

where $f$ and $g$ are random induced Huctuations due to the free turbulence. The function $g$ is related to the vorticity fluctuations in the flow. Equations (18) show that the axial perturbation $(u)$, pressure perturbation $(p)$ and density perturbation $(\rho)$, that are of order of the shock strength $-\left(\epsilon^{2 / 3}\right)$, interact with the transverse velocity perturbations $v_{1}$ and $w_{1}$. that are of a smaller scale $(\epsilon)$.

The substitution of $u=g / A+\bar{u}$ in $(18 \mathrm{c}),(18 \mathrm{~d})$ and (18e) results in a problem for solving a velocity potential function $o\left(x^{*}, y, z, t^{*}\right)$ where:

$$
\begin{aligned}
& \bar{u}=\frac{\partial \phi}{\partial x^{*}}, \quad v_{1}=\frac{\partial \phi}{\partial y}, \quad u_{1}=\frac{\partial \phi}{\partial z} . \quad p=-\gamma \frac{\partial \phi}{\partial x^{*}} \\
& 2 \phi_{x^{*} t^{*}}+\left(K^{*}+\frac{g}{\gamma}+f+(\gamma+1){\phi_{x}}^{*}\right){O_{x}{ }^{*} x^{*}}-\left(O_{y y}+\phi_{z:}\right)=-\frac{1}{\gamma} \frac{\partial g}{\partial t^{*}}
\end{aligned}
$$

In a conservative form $\mathrm{Eq} .(19 \mathrm{~b})$ is given by:

$$
\left.\left(2 \phi_{x^{*}}+\frac{1}{\gamma} g\right)_{\ell^{\bullet}}+\left((K+g / \gamma+f) \phi_{z} \cdot+(\gamma+1) \phi_{x}^{2} \cdot / 2\right)\right)_{x^{*}}-\left(\phi_{y}\right)_{y}-\left(\phi_{z}\right)_{z}=0
$$

The exact shock jump conditions (Ref. 32) must be satisfied along any shock surface $x^{*}-h\left(y, z, t^{*}\right)=0$ that may appear in the solution. To the leading orders they result in:

$$
[f]=0 . \quad[g]=0
$$




$$
\begin{gathered}
-2\left[\phi_{x^{*}}\right] \frac{\partial h}{\partial t^{*}}+\left(K^{*}+g+f\right)\left[\phi_{x^{*}}\right]+(\gamma+1)\left[\frac{\phi_{x}^{2}}{2}\right]+\left[\phi_{y}\right] \frac{\partial h}{\partial y}+\left[\phi_{:}\right] \frac{\partial h}{\partial z}=0(20 \mathrm{~b}) \\
{\left[\phi_{y}\right]+\left[\phi_{x} \cdot\right] \frac{\partial h}{\partial y}=0 \quad\left[\phi_{z}\right]+\left[\phi_{x} \cdot\right] \frac{\partial h}{\partial z}=0}
\end{gathered}
$$

where $[a]$ represents the jump across the shock property $a,[a]=a_{B}-a_{A}$. Equations (20a) show that to the leading order there is no jump in entropy across the shock, $[S]=0$. Equations (11) and (1S) also show that the local Mach number $W_{e}$ at any point in the flow is given by:

$$
M_{\ell}^{2}-1=\epsilon^{2 / 3} u^{*} . \quad u^{*}=\left\{(\gamma+1) \phi_{\nu} \cdot+K^{*}+f+\frac{g}{\gamma}\right\}
$$

The flow is locally supersonic when $(\gamma+1) \phi_{x^{*}}+K+f+\frac{q}{\gamma}>0$, sonic when $(\gamma+1) \phi_{x^{*}}+h^{-}+f+\frac{q}{\gamma}=0$, and subsonic when $(\gamma+1) \phi_{x^{*}}+h^{*}+f+\frac{q}{\gamma}<0$. Equations (19) and (20) are an extended version of the classic unsteady small-disturbance equation for transonic aerodynamics (Cole and Cook ${ }^{33}$ ). The only changes are due to the random terms $g$ and $f$. Starting from given functions for $f$ and $g$ and initial conditions that describe a given basic shock, Eqs. (19) and (20) can be integrated in space and time to describe the development of the shock wave and pressure field behind it. A numerical algorithm to solve these equations is described in Section 4.

An alternative approach may be found by taking a $x^{*}$ - derivative of (18c) and using Eqs. (18a) and (14). The pressure perturbation ( $p$ ) satisfies the equation:

$$
\frac{\partial}{\partial x^{*}}\left(\frac{\partial p}{\partial t^{*}}+\frac{\hbar+f+g / \gamma}{2}-\frac{\gamma+1}{2 \gamma} p \frac{\partial p}{\partial x^{*}}\right)=\frac{1}{2}\left(\frac{\partial^{2} p}{\partial y^{2}}+\frac{\partial^{2} p}{\partial z^{2}}\right)
$$

Equation (22) is an extended version of the classic $\mathrm{KKZ}$ equation that describes the propagation of nonlinear sound beams with narrow angular spectrum in an inviscid fluid (Zabolotskaya et al. ${ }^{30}$. Kuznetsov ${ }^{31}$ ). Eq. (22) also has a similar form to the model equation that has been recently developed by Pierce $^{17}$ using logical considerations only. 
Equations (19) and (22) show that diffraction effects. nonlinear steepening, focusing and caustic effects and random induced fluctuations due to turbulence interact simultaneously to determine the development of the shock wave in space and time and the pressure field behind it. Turbulence tends to change the local speed of sound in the flow across the shock and through this effect to reduce or to magnify the strength of the jump along the basic shock (see Eq. (21)) or to distort the shock front. These changes may result in unsteady motion of the shock front or in caustic vertices or in reflected shocks behind the incident wave that can produce the variety of pressure signatures of sonic booms that are measured in experiments.

\section{Finite Difference Scheme}

A finite difference algorithm to solve the unsteady mixed-type elliptichyperbolic flow around the shock wave has been developed. Murman and Cole $^{34}$ and Cole and Cook $^{33}$ techniques are used. A fully conservative scheme that is based on the conservative form (Eq. 19c) is used. In this way the difference equations also contain the shock relations (Eqs. (20)).

Consider a uniform finite difference mesh $\left(\Delta x^{*} . \Delta y, \Delta z, \Delta t^{*}\right)$ in space and time, with points $\left(x^{*}, y, z, t^{*}\right)$ labeled by $(i, j, k, n)$. The results can be easily generalized to a variable mesh. Eq. (19c) can be expressed in a conservative flux form for a box centered on a mesh point $(i, j, k)$. Therefore,

$$
\begin{gathered}
\frac{1}{\Delta t^{*}}\left\{\left(2 \phi_{x^{*}}+\frac{1}{\gamma} g\right)_{(i, j, k, n)}-\left(2 \phi_{x^{*}}+\frac{1}{\gamma} g\right)_{(i, j, k, n-1)}\right\}+ \\
+\frac{1}{\Delta x^{*}}\left\{\left(\left(K+\frac{1}{\gamma} g+f\right) \phi_{x^{*}}+(\gamma+1) \phi_{x^{*}}^{2} / 2\right)_{\left(i+\frac{1}{2}, j, k, n\right)}\right. \\
\left.-\left(\left(K+\frac{1}{\gamma} g+f\right) \dot{\phi}_{x} \cdot+(\gamma+1) \phi_{x}^{2} \cdot / 2\right)_{\left(i-\frac{1}{2}, j, k, n\right)}\right\} \\
-\frac{1}{\Delta y}\left\{\left(\phi_{y}\right)_{\left(i, j+\frac{1}{2}, k, n\right)}-\left(\phi_{y}\right)_{\left(i, j-\frac{1}{2}, k, n\right)}\right\}-\frac{1}{\Delta z}\left\{\left(\phi_{z}\right)_{\left(i, j, k+\frac{1}{2}, n\right)}-\left(\phi_{z}\right)_{\left(i, j, k-\frac{1}{2}, n\right)}\right\}=0
\end{gathered}
$$


$\left(\phi_{y}\right)$ and $\left(\phi_{z}\right)$ are always calculated from a centered expression. However. the approximation of $\left(\phi_{\boldsymbol{r}}\right)$ strongly depends on whether locally, at a point. the flow is subsonic, supersonic, sonic or it is a shock point. Extending References 33, 34 methodologies to our case and using Eq. (21), a centered approximation and a backward expression are given for $u^{*}$ :

$$
\begin{aligned}
u_{(i, j, k, n)}^{-c}=K^{*} & +f(j, k, n)+\frac{1}{\gamma} g(j, k, n) \\
& +\frac{\gamma+1}{2 \Delta x^{*}}(\phi(i+1, j, k, n)-\phi(i-1, j, k, n)) \\
u_{(i, j, k, n)}^{=b}=K^{-} & +f(j, k, n)+\frac{1}{\gamma} g(j, k, n) \\
& +\frac{\gamma+1}{2 \Delta x^{*}}(\phi(i, j, k, n)-\phi(i-2, j, k, n))
\end{aligned}
$$

The local type of the flow is determined by the following table: ${ }^{33.34}$

\begin{tabular}{|c|c|c|r|}
\hline condition & $u^{* c}$ & $u^{* b}$ & local flow is \\
\hline 1 & $<0$ & $<0$ & subsonic \\
2 & $>0$ & $>0$ & supersonic \\
3 & $>0$ & $<0$ & a sonic point \\
4 & $<0$ & $>0$ & a shock point \\
\hline
\end{tabular}

Table 1

Eq. (23) is developed in a specific form according to the local type of the flow. When the flow is locally subsonic, an elliptic difference form is used: 


$$
\begin{aligned}
& G(i, j, k, n)=\frac{1}{\Delta x^{*} \Delta t^{*}}(\phi(i+1, j, k \cdot n)-o(i-1 . j, k, n-1)) \\
& -\frac{2}{\Delta t^{*}} \phi_{x^{*}}(i, j, k, n-1)+\frac{1}{\gamma \Delta t^{*}}(g(j, k, n)-g(j, k, n-1)) \\
& +\left\{K+f(j \cdot k \cdot n)+\frac{1}{y} g(j \cdot k \cdot n)\right. \\
& \left.+\frac{\gamma+1}{2 \Delta x^{*}}(o(i+1, j, k, n)-\phi(i-1, j, k, n))\right\} \\
& \frac{o(i+1, j, k, n)-2 o(i, j, k, n)+o(i-1, j, k, n)}{\left(\Delta \cdot c^{*}\right)^{2}} \\
& -\frac{1}{(\Delta y)^{2}}(0(i, j+1, k, n)-20(i, j, k . n)+o(i . j-1, k, n)) \\
& -\frac{1}{(\Delta z)^{2}}(\phi(i, j, k+1 . n)-2 \phi(i . j, k, n)+\phi(i, j, k-1, n))=0
\end{aligned}
$$

When the flow is locally supersonic, a hyperbolic difference form is used:

$$
\begin{aligned}
G(i, j, k, n)= & \frac{2}{\Delta x^{*} \Delta t^{*}}(\phi(i, j, k, n)-\phi(i-1 . j, k, n))- \\
& -\frac{2}{\Delta t^{*}} \phi_{x} \cdot(i . j, k, n-1)+\frac{1}{\gamma \Delta t^{*}}(g(i, j, k, n)-g(i, j, k, n-1))
\end{aligned}
$$




$$
\begin{aligned}
& +\left\{K+f(j, k, n)+\frac{1}{\gamma} g(j, k, n)\right. \\
& \left.+\frac{\gamma+1}{2 \Delta x^{*}}(\phi(i, j, k, n)-\phi(i-2, j, k, n))\right\} \\
& . \frac{\phi(i, j, k, n)-2 \phi(i-1, j, k, n)+\phi(i-2, j, k, n)}{\left(\Delta x^{*}\right)^{2}} \\
& -\frac{1}{(\Delta y)^{2}}(\phi(i, j+1, k, n)-2 \phi(i, j, k, n)+\phi(i, j-1, k, n)) \\
& -\frac{1}{(\Delta z)^{2}}(\phi(i, j \cdot k+1, n)-2 \phi(i, j, k, n)+\phi(i, j, k-1, n))=0 .
\end{aligned}
$$

When the flow is locally sonic. $(\gamma+1) 0_{r} \cdot H+f+\frac{1}{\gamma} g=0$. Then the sonic point difference form is:

$$
\begin{aligned}
G(i, j, k, n)= & \frac{1}{(\Delta y)^{2}}(\phi(i, j+1, k, n)-20(i, j, k, n)+o(i, j-1, k, n)) \\
& +\frac{1}{(\Delta z)^{2}}(\phi(i, j, k+1, n)-2 o(i, j, k, n)+\phi(i, j, k-1, n)) \\
& -\frac{1}{(\gamma+1) \Delta t^{*}}\left(\frac{\gamma-1}{i}(g(j, k, n)-g(j, k, n-1))\right. \\
& -2(f(j, k, n)-f(j, k, n-1)))=0 .
\end{aligned}
$$

When locally there is a shock point, a shock point difference operator is used where the flux ahead of the shock may be approximated by a backward 
formula and the flux behind the shock by a centered formula.

$$
\begin{aligned}
& G(i, j, k, n)=\frac{1}{\Delta x^{*} \Delta t^{*}}(o(i+1 . j \cdot k \cdot n)-o(i-1 . j \cdot k \cdot n)) \\
& -\frac{2}{\Delta t^{*}} \mathcal{O}_{r} \cdot(i, j, k, n-1)+\frac{1}{; \Delta t^{*}}(g(j, k, n)-g(j, k, n-1)) \\
& +\left\{K+f(j \cdot k, n)+\frac{1}{\gamma} g(j, k, n)\right. \\
& +\frac{\gamma+1}{2 \Delta \cdot x^{*}}(o(i+1, j \cdot k \cdot n)-o(1, j \cdot k \cdot n)+\phi(i-1 \cdot j, k \cdot n) \\
& +o(i-2 . j, k \cdot n))\} \\
& \frac{\phi(i+1, j, k \cdot n)-\phi(i, j . k . n)-\phi(i-1, j, k \cdot n)+\phi(i-2, j, k, n)}{\left(\Delta x^{*}\right)^{2}} \\
& -\frac{1}{(\Delta y)^{2}}(o(i . j+1 . k, n)-20(i . j, k, n)+\phi(i, j-1, k, n))- \\
& -\frac{1}{(\Delta z)^{2}}(o(i . j \cdot k+1 . n)-2 o(i . j \cdot k \cdot n)+\phi(i, j, k-1, n))=0 \text {. }
\end{aligned}
$$

Starting from initial conditions that describe a given shock wave in the space for $t=0$ (or $n=0$ ), and given the functions $f(y, z, t)$ and $g(y, z, t)$, equations (25) through (28) can be applied for $n=1$ at any mesh point according to Table 1 . They can be solved by an iterative point or line or plane - relaxation algorithm until at any point $\max |G(i, j, k, 1)|<\delta$ where $\delta$ is a given small tolerance of convergence. Then $\phi_{x^{*}}(i, j, k, 1)$ can be calculated at any mesh point and the process is restarted for the next time step. In this way the shock motion and pressure field behind it can be integrated in space and time and the effect of various deterministic and 
random tluctuations $f$ and $g$ can be studied. Numerical calculations of various examples are underway and will be presented in a future report.

\section{Conclusions}

The review of the theoretical studies of the interaction of shock waves with free stream vortical flows or turbulence shows that this complex nonlinear interaction is still an open problem to analyze. The analysis of the linearized problem of the interaction of a weak shock with relatively small disturbances shows that it is an invalid approach when the perturbations are of the order of shock strength. However, in a coordinate system moving with the basic weak shock, the problem may fit the transonic theory framework.

A new transonic small-disturbance model has been developed where a rescaling of the axial coordinate and time has been considered to capture the basic nonlinear effects that occur in the flow across the shock. This model results in two alternative approaches: (1) an equation for solving a velocity potential function that is described by an extended version of the classic small-disturbance equation for unsteady transonic aerodynamics; ${ }^{33}$ and (2) a nonlinear equation to describe the pressure field that is similar to the model equation recently presented by Pierce $^{17}$ using logical considerations only. This equation also has extended form of the classic equation that describes the propagation of nonlinear sound beams with narrow angular spectrum. . $^{30.31}$

Both approaches show that diffraction effects, nonlinear steepening, focusing and caustic effects and random induced turbulence fluctuations interact simultaneously to determine the development of the shock wave in space and time and the pressure field behind it. Turbulence fluctuations tend to change the local speed of sound in the flow across the shock and through this effect to reduce or magnify the strength of the basic shock.

A finite difference scheme that uses Murman and Cole ${ }^{34}$ finite-difference 
techniques for solving mixed-type elliptic-hyperbolic flows with shock waves has also been presented. Numerical calculations of the interaction of shock waves with various deterministic and random fluctuations will be presented in a future report. We will also look for analytical methods to identify the basic relations between given turbulence properties and the development of the shock waves and the pressure field behind it. It is expected to find solutions that will describe both peaked or rounded or messy pressure signatures as were recorded in experiments. We also intend to extend the model to include humidity and winds effects that are also known to have a significant effect on sonic boom pressure peaks and rise times.

\section{Acknowledgements}

This work was carried out with the support of NASA Langley Research Center under $A$ ward NAG-1-1362. The authors would like to acknowledge Dr. G. L. McAninch for funding and monitoring this research.

\section{References}

[1] Hubbard, H. H. . Maglieri. D. J., Huckel. V. and Hilton, D. A., "Ground Measurements of Sonic Boom Pressures for the Altitude Range of 10,000 to 75,000 feet," NASA TR R-198, July 1964.

[2] Maglieri, D. J., "Sonic Boom Flight Research - Some Effects of Airplane Operations and the Atmosphere on Sonic Boom Signatures," in Sonic Boom Research, Edited by Seebass, A. R., NASA SP-147, April 1967.

[3] Garrick, I. E. and Maglieri, D. J., "A Summary of Results on Sonic Boom Pressure-Signature Variations Associated with Atmospheric Conditions," N.ASA TN D-4588. May 1968. 
[4] Downing, M. J., "Lateral Spread of Sonic Boom Measurements from U.S. Air Force Boomfile Flight Tests." N.ASA CP-31i2. Feb. 1992, pp. $117-135$.

[5] Willshire, Jr., W. L. and Devilbiss, D. W.. "Preliminary Results from the White Sands . Missile Range Sonic Boom Propagation Experiment," NASA CP-31i2, Feb. 1992, pp. 137-149.

[6] Lipkens, B. and Blackstock, D.T.. ".Model Experiment to Study the Effect of Turbulence on Risetime and Waveform of $N$ Waves." NASA C'P-3172, Feb. 1992. pp. 9T-10 7 .

[7] Leatherwood. J. D. and Sullivan. B. .M., "Subjective Loudness response to Simulated Sonic Booms," NASA CP-3172. Feb. 1992. pp. 151-170.

[8] Crow, S. C.. "Distortion of Sonic Bangs by Atmospheric Turbulence," J. Fluid Mechanics, vol. 37. part 3. 1969. pp. 529-563.

[9] Plotkin. K. J. and George. A. R.. "Propagation of Weak Shock Waves Through Turbulence," J. Fluid Mechanics, vol. 54, part 3, 1972, pp. $449-46 \mathrm{~T}$.

[10] Ffowcs Williams. J. E. and Howe. M. S.. "On the Possibility of Turbulent Thickening of Weak Shock Waves." J. Fluid Mechanics, vol. 58, part 3, 19i3. pp. 461-480.

[11] Pierce, A. D., "Spikes on Sonic-Boom Pressure Waveforms," J. Acoustical Society of America. vol. 44. 110. 4. 1968. pp. 1052-1061.

[12] Pierce, A. D., "Statistical Theory of Atmospheric Turbulence Effects on Sonic-Boom Rise Times." J. Acoustical Society of America, vol. 49, no. 3 (part 2), 1971, pp. 906-924.

[13] Cramer, M. S. and Seebass, A. R., "Focusing of Weak Shock Waves at an Arête," J. Fluid Mechanics. vol. S8, part 2, 1978. pp. 209-222.

[14] Gill, M. and Seebass. A. R.: "Nonlinear Acoustic Behavior at a Caustic: An Approximate Analytical Solution." AI.A Paper 73-1037, AIA.A Aeroacoustics Conference. Seattle. Wash.. 1973. 
[15] Sturtevant. B. and Kulkarny, V. A.. "The Focusing of Weak Shock Waves," J. Fluid Mechanics, vol. i3. 1976. pp. 651-6i1.

[16] Sparrow, V. W. and Pierce, A. D.. "Simulations of Sonic Boom Ray Tube Area Fluctuations for Propagation Through Atmospheric Turbulence Including Caustics via a Monte Carlo Method." N.ASA CP-3172, Feb. 1992. pp. $49-62$.

[17] Pierce, A. D., "Wave Equations and Computational Models for Sonic Boom Propagation Through a Turbulent Atmosphere." NASA CP. 3172. Feb. 1992. pp. 31-48.

[18] Hollingsworth. .I. A. and Richards. E. J.. "A Schlieren Study of the Interaction Between a Vortex and a Shock Wave in a Shock Tube," A.R.C. Rept. 17985, F.Y12323, 1955.

[19] Dosanjh. D. S. and Weeks. T. M.. "Interaction of a Starting Vortex as Well as a Vortex Street with a Traveling Shock Wave," AIAA J., vol. 3. 1965. pp. 216-223.

[20] Naumann, A. and Hermanns, E.. "On the Interaction Between a Shock Wave and a Vortex Field," in Noise Mechanisms, AGARD CP-131, 1973, pp. 23-1.

[21] Ribner. H. S.. "Spectra of Noise and Amplified Turbulence Emanating from Shock-Turbulence Interaction." AIAA J., vol. 25. 198\%. pp. 436 .

[22] Kovasnay, L. S. G.. "Turbulence in Supersonic Flow," Journal of the Aeronautical Sciences. vol. 20, 1953, pp. 657-674.

[23] Moore, F. K., "Unsteady Oblique Interaction of a Shock Wave with a Plane Disturbance," VACA Report 1165. 1954.

[24] Ribner. H. S., "Shock-Turbulence Interaction and the Generation of Noise," NACA Report 1233. 1955.

[25] Chang, C. T., "Interaction of a Plane Shock and Oblique Plane Disturbances with Special Reference to Entropy Waves," Journal of the Aeronautical Sciences. vol. 24. 1957. pp. 675-682. 
[26] Ribner, H. S., "Cylindrical Sound Wave Generated by Shock-Vortex Interaction," AIAA J.. vol. 23, no. 11, 1385. pp. 1708-1715.

[27] Zang, T. A., Hussaini. M. Y. and Bushnell, D. .M., "Numerical Computations of Turbulence Amplification in Shock- Wave Interactions." AIAA J., vol. 22, no. 1. 1984, pp. 13-21.

[28] Meadows, K. R., Kumar. A. and Hussaini, M. Y., "Computational Study on the Interaction Between a Vortex and a Shock Wave," $A I A A$ $J$. , vol. 29, no. 2. 1991. pp. 174-1i9.

[29] Ellzey, J. L.. Picone. M. and Oran. E. S.. "The Interaction of a Shock with a Compressible Vortex." Naral Research Laboratory M.R. 6919, 1992.

[30] Zabolotskaya, E.A. and Khorkhlov, R.V.. Akust. Zhurnal 15. 1. 1969. pp. 40.

[31] Kuznetsov. V. P.. Akust. Zhurnal 6. 4. 1970. pp. 548.

[32] Courant, R. and Friedrichs. K. O.. "Supersonic Flow and Shock Waves," Interscience Publishers. Inc. N.Y.. pp. 297-302.

[33] Cole, J. D. and Cook. L. P., Transonic Aerodynamics, North-Holland, 1986.

[34] Murman. E. M. and Cole. J. D.. "Calculation of Plane Steady Transonic Flows," AIAA J.. vol. 9, pp. 114-121. 
